\title{
AN EXTENSIVE CASE OF PLANTAR WARTS
}

RICHARD L. SUTTON, M.D. KANSAS CITY, mo.

The symptomatology of verruca plantaris, a puzzling but comparatively common disease of the skin, was first described by Gorju, ${ }^{1}$ of Paris, over half a century ago. More recently the disorder has been carefully studied by Dubreuilh ${ }^{2}$, Montgomery ${ }^{3}$, Bowen ${ }^{4}$ and others, but, despite the publicity given it by these contributors, the true nature of the condition is seldom recognized even to-day.

For this reason the following example. which is one of the most extensive I have seen, may be of general interest:

L. C.. single woman aged 20 , student, referred to me by Dr. Jabez N. Jackson of this city, was a native of Massachusetts and a resident of Boston. Four years previously she

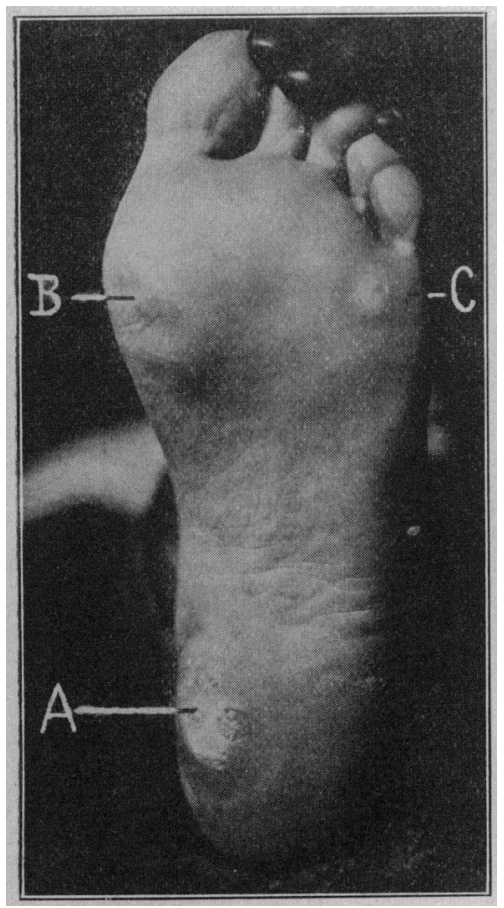

Fig. 1. The epldermal "lid" has been removed at $A$, disclosing a group of five verrucae. The callus-like masses at $B$ and $C$ both contain typical plantar warts.

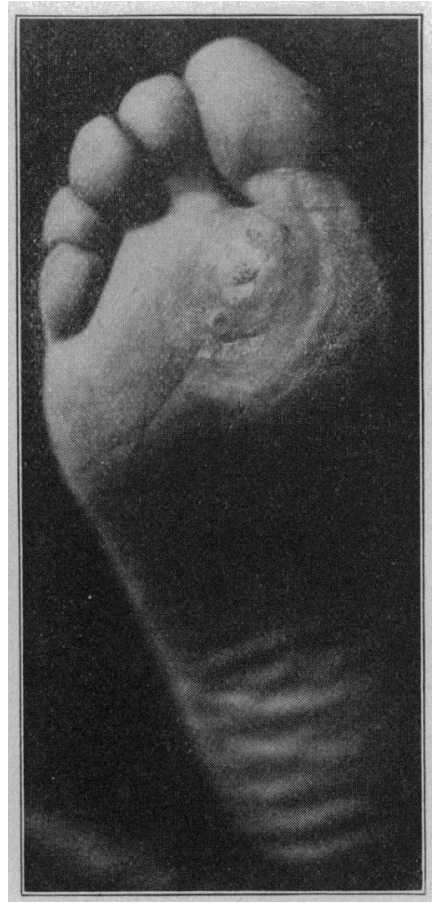

Fig. 2.-Group of plantar warts on right foot. The cov ering has been removed from the one in the middle. single group of three lesions, arranged in a row, near the base of the great toe (Fig. 2 ).

The middle and oldest of the three vas simply a yellowish, callus-like mass, but the other two were smaller and sharply circumscribed, with slightly elevated borders and brownish, tender centers.

When the outer layers of epidermis covering the lesions was removed by means of salicylic acid plaster, the typical, well-like cavities, partially filled with tough, stringy, opaque masses of transformed epithelium, were exposed. For laboratory study one of the lesions on the right foot and a portion of the large one on the left heel were excised with a sharp cutaneous punch. The histologic characteristics of the growths could best be studied in the specimen from the right foot (Figs. 3 and 4 ). The larger portion of the tumor consisted of imperfectly keratinized horny material. With the exception of a pronounced degree of papillary hypertrophy in the vicinity of the growth the corium was little changed. The border of the lesion was acanthotic, and many of the cells here and in the center of the area contained vacuoles. Both granular and horny layers were much thickened. The peculiar, small, round, highly refractile, protozoa-like, intercellular bodies which Bowen first described were

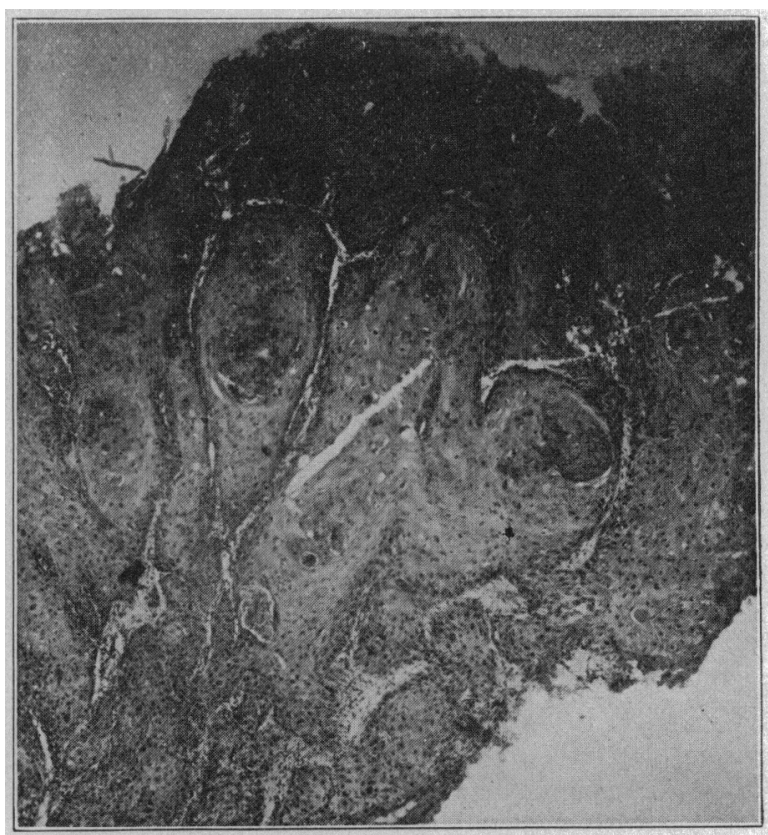

Fig. 3.-Plantar wart from right foot, showing general structure (low magnification). had been troubled with a "soft corn" on the sole of her left foot, and at one time since then a college room-mate had had several growths on the bottoms of her feet similar to those from which the pationt sought relief.

At first glance the lesions resembled large, oval callosities. The most troublesome one was situated near the inner border of tha left heel, and measured 2 by $6 \mathrm{~cm}$. (Fig. 1, $A$ ). The overlying epidermis was smooth and translucent and a superficial examination revealed no hint of the presence of verrucae. On deep pressure, however, the tumor was found to be exceedingly tender at several different points. Just posterior to the fifth metatarsophalangeal articulation was a similar but somewhat smaller growth (Fig. $1, C$ ), which also was very sensitive to pressure, and near the inner side of the ball of the foot was a third (Fig. 1, B). The right sole presented a

1. Gorju : Thèse de Paris, 1857.

2. Dubreuilh: Ann. de dermat. et de syph., 1895, p. 441.

3. Montgomery, D. W.: Papilloma of the Sole, Trf Jourval A. M. A., July 11, 1903 , p. 100.

4. Bowen: Boston Med. and Surg. Jour., 1907, p. 781 present in considerable numbers. As he has stated, they probably represent some form of nuclear degeneration or alteration.

Plantar warts are notoriously resistant to treatment. The therapeutic measures usually employed in combating the disease elsewhere on the body often prove ineffectual here, and recurrence occasionally takes place even after excision.

Of all the methods tried, I should place Pusey's carbon dioxid snow first in value, with fulguration second and the Roentgen rays third. Before the snow or the electric current is applied the epidermal "lids" of the little tumors should be removed by means of a 10 per cent. salicylic acid plaster. Roentgenotherapy is particularly applicable in those cases presenting numerous lesions, and often following its use the growths disappear as if by magic. 
In the present instance the patient was exceedingly anxious to get well as quickly as possible, and all three methods were tried, the result being an apparently complete cure at the end of about four weeks.

613-614 Commerce Building.

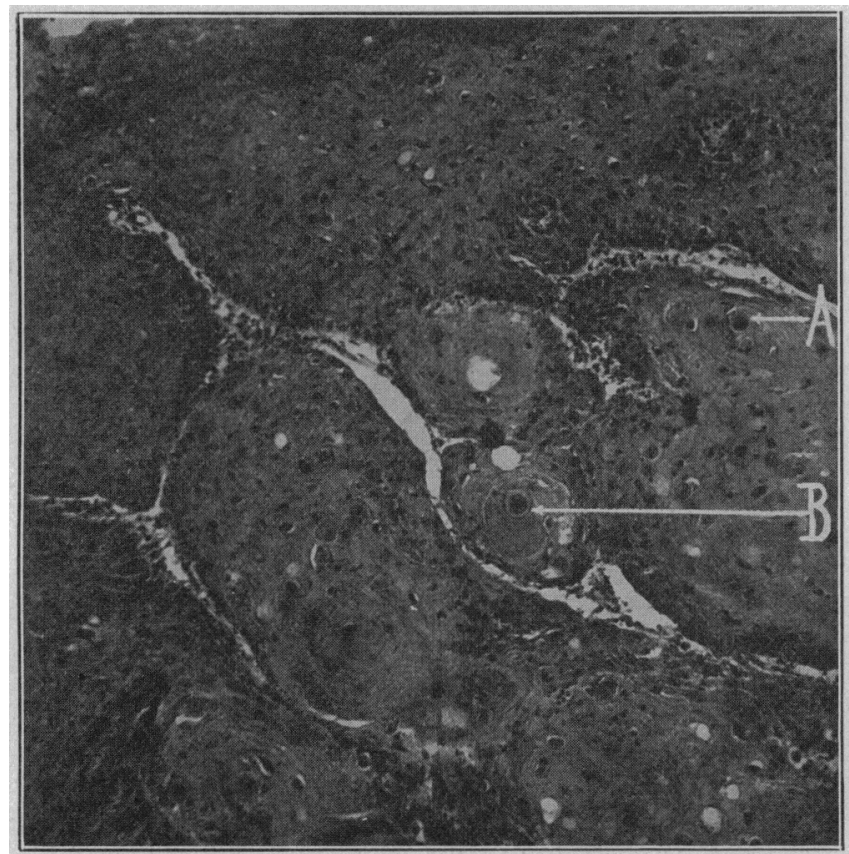

Fig. 4.-Flantar wart, showing epithelial columns, with degenrated cellular changes and "ballooning." Protozoa-like bodies at $A$ and $B$ (moderate magnification).

\section{CANCER OF THE SPLENIC FLEXURE}

\section{P. E. TRUESDALE, M.D.}

FALL RIVER, MASS.

The splenic flexure marks an intermediate position in the colon both anatomically and physiologically. The proximal segment, the cecum and the ascending and transverse colon, as observed by Cannon, ${ }^{\mathbf{1}}$ churn and mix the bowel contents until the moisture is largely removed. Starling ${ }^{2}$ has compared this process with the contractions in the pyloric end of the stomach. The dry part of the intestinal mass begins to collect, therefore, at the splenic flexure, where there is frequently a sharp angulation in the colonic tract. This abrupt change in the course of the bowel causes a hindrance to the passage of its contents, resulting in one of the pressure-points in the colon. The predisposing causes of carcinoma, ulceration and chronic irritation exist here, no doubt, as in regions in which cancer is more frequent. A routine examination of this territory when the abdomen is opened reveals the fact that the costocolic ligament, an embryologic structure reduplicating the peritoneum from the omentum, varies considerably in its appearance and density, enough so to convince one that inflammatory changes here are more common than is generally believed. In spite of this, however, cancer at the splenic flexure is relatively rare, developing slowly and metastasizing late.

The lymphatic vessels and glands of this region are so related that the conditions for the spread of carcinoma are not favorable. Madeling ${ }^{3}$ reports seven cases

1. Cannon : Am. Jour. Physiol., 1901, vi, 251

1. Starling: The Physiology of Digestion, p. 146.

. Madeling : Arch. f. klin. Chir., Ixxxi, 1 . of his own and ninety-three from the literature, from which he draws the important conclusion that cancer of the splenic flexure is curable by intestinal resection even in the advanced stages of the disease. Mayo ${ }^{4}$ reports seven cases of cancer of the transverse colon, including the hepatic and splenic flexures. There was one operative death. Two patients were alive and well after one year. One was alive and well three years after operation. One could not be traced.

The following two cases of cancer at the splenic flexure presented a somewhat striking difference in the clinical picture:

CASE 1.-M. B., aged 63, housewife, native born (seen in consultation with Dr. George W. Parlow), was admitted to the Highland Hospital Nov. 5, 1912, for intestinal obstruction of three days' duration. For several weeks she had been troubled with unusual sluggishness of the bowels, associated with anorexia, distress after meals and a perceptible loss in weight. Vomiting began three days before admission, and distention of the abdomen steadily increased. Cathartics and enemas had failed. On examination the abdomen was distended but not tense. Deep palpation revealed no tumor. The abdomen was opened by a left rectus incision. The small bowel was considerably distended, the cecum enormously so. The descending colon was collapsed. Well up under the left costal arch was a tumor involving the splenic flexure of the colon. After mobilization of the bowel at the tumor site, a preliminary colostomy was made by bringing the transverse colon through the upper angle of the incision. November 25 , a resection of the splenic flexure was done. The transverse and descending colon were freely mobilized. A division of the transverse colon was made adjoining the colostomy opening. The splenic flexure was then resected with a good margin of healthy bowel.

A lateral anastomosis was made after a method described by Bloodgood," wherein the closed end of each segment was sutured outside of the parietal peritoneum in the line of incision, thereby placing extraperitoneally the points of suture in which the danger of leakage is greatest. This safety measure was appreciated during the convalescence of this patient. The pathologic examination of the specimen removed showed it to be adenocarcinoma. December 15 , there was a slight

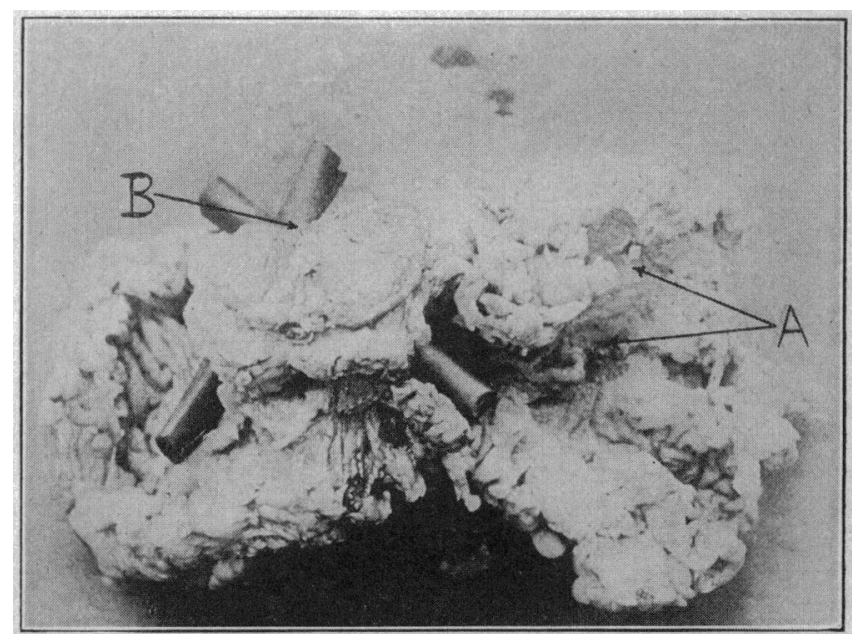

Fig. 1 (Case 1).-Segment of colon removed at operation showing (A) annular adenocarcinoma which had occluded the lumen at the splenic flexure, and $(B)$ colostomy opening in the transverse colon.

discharge of fecal matter from the wound. This gradually cleared up and the patient was discharged in good condition, Jan. 1, 1913. This patient presented herself for examination in February, 1914. During the year following her operation

4. Mayo: Tr. Am. Surg. Assn., 1909, xxvii, 338.

5. Bloodgood: Tr. Am. Surg. Assn., 1909, xxvii, 340. 